This is the peer reviewed version of the following article: He Xuezhong and Shi Lei 2012, 'Boundedly rational equilibrium and risk premium', Blackwell Publishing, vol. 52, no. 1, pp. 71-93. which has been published in final form at http://dx.doi.org/10.1111/j.1467-629X.2011.00421.x This article may be used for non-commercial purposes in accordance With Wiley Terms and Conditions for self-archiving' 


\title{
BOUNDEDLY RATIONAL EQUILIBRIUM AND RISK PREMIUM
}

\author{
XUE-ZHONG HE AND LEI SHI \\ School of Finance and Economics \\ University of Technology, Sydney \\ PO Box 123 Broadway \\ NSW 2007, Australia
}

\begin{abstract}
When people agree to disagree, how the disagreement among agents impact on the market is the main concern of this paper. With the standard mean variance framework, this paper considers a market of two risky assets and two agents who have different preference and biased beliefs in the mean and variance/covariance of the asset returns. By constructing a consensus belief, the paper develops an concept of boundedly rational equilibrium (BRE) to characterize the market equilibrium and examines explicitly the impact of heterogeneity on the market equilibrium and risk premium when the disagreements among the two agents are mean preserved spreads of a benchmark homogeneoue belief. It shows that, in market equilibrium, the biased mean preserved spreads in beliefs among the two agents have significant impact on the risk premium of the risky assets and market portfolio, and adding a riskless asset in the market magnifies the impact of the heterogeneity on the market. The results show that both optimism/pessemism and confidence/doubt can increase the market risk premium and reduce the riskfree rate.
\end{abstract}

JEL Classification: G12, D84.

Keywords: Asset prices; biased beliefs; boundedly rational equilibrium; zero-beta CAPM; equity premium and risk-free rate puzzles.

Date: Preliminary Version: July 2009; Current version: September 4, 2009. Financial supports from the Australian Research Council (ARC) under Discovery Grant (DP0773776) and a Faculty Research Grant at UTS are gratefully acknowledged. 


\section{INTRODUCTION}

To better explain market anomalies, puzzles and various market phenomena, economics and finance are witnessing an important paradigm shift, from a representative, rational agent approach towards a behavioral, agent-based approach in which economy and markets are populated with bounded rational agents who have heterogeneous beliefs. When the heterogeneity in beliefs is not due to asymmetric information but rather to intrinsic differences in how to view the world, people agree to disagree. The heterogeneity in beliefs among agents are very often characterized by notions of optimism and pessimism, overconfidence and doubt. Literatures have made a significant contribution to the understanding of the market aggregation when agents differ on their expectations and the impact of heterogeneous beliefs amongst investors on market equilibrium, see, for example, Lintner (1969), Rubinstein (1976), Williams (1977), Abel (1989, 2002), Detemple and Murthy (1994), Zapatero (1998)), and more recently Calvet, Grandmont and Lemaire (2004), Wenzelburger (2004), Böhm and Chiarella (2005), Böhm and Wenzelburger (2005), Jouni and Napp (2006), Sharpe (2007), Gollier (2007), Chiarella, Deici and He (2006, 2009) and Horst and Wenzelburger (2008).

The notion of overconfidence has been explored in finance literature (see, e.g., DeLong, Shleifer, Summers and Waldmann (1990), Kyle and Wang (1997), and Daniel, Hirshleifer and Subrahmanyam (1998)). Several empirical studies of professionals' economic forecasts and psychological surveys indicate that agents have optimism and overconfidence $^{1}$ about their own (relative) abilities (see, e.g., Rabin (1998), Hirshleifer (2001), and Giordani and Soderlind (2006)). One of the driving forces for the development of the literature in heterogeneous beliefs is to explain equity premium and risk-free rate puzzles (see Mehra and Prescott (1985) and Weil (1989)), to which several theoretic explanation have been proposed recently. For example, Barberis, Huang and Santos (2001) adopt a non-standard utility function, motivated by prospect theory; Benartzi and Thaler (1995) consider myopic loss aversion. Deviating from rational expectation in the standard neoclassical paradigm, when beliefs are exogenously given, it has been found (see, e.g., Detemple and Murthy (1994), Abel (2002), Calvet et al. (2004), Jouini and Napp (2006), and Gollier (2007)) that a pessimistic bias and

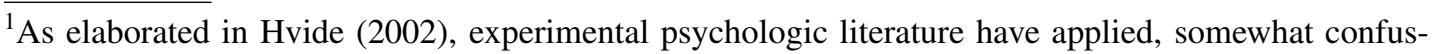
ingly, two distinct meanings of the term 'overconfidence', overconfidence ${ }_{1}$ and overconfidence (called $^{2}$ in Hvide (2002)). In the stock market, overconfidence ${ }_{1}$ relates to a skewed first moment of a subjective probability distribution, while overconfidence ${ }_{2}$ relates to a skewed second moment of a subjective probability distribution. There is no clear relation between overconfidence ${ }_{1}$ and overconfidence ${ }_{2}$ since they reflect different underlying phenomena. In Abel (2002), a uniform pessimism is defined as (the subjective distribution being) a leftward translation of the objective distribution, doubt as a meanpreserving spread of the objective distribution. To avoid confusion, in our discussion, we adopt the notions of Abel (2002) and refer overconfidence ${ }_{1}$ and overconfidence ${ }_{2}$ to optimism and overconfidence, respectively. The confidence in DeLong et al. (1990) and Kyle and Wang (1997) is actually referred to overconfidence $_{2}$.
} 
doubt in the subjective distribution of the growth rate of consumption and a positive correlation between risk tolerance and pessimism (doubt) leads to an increase of the market price of risk. To discipline the heterogeneity in beliefs and to understand how agents form their beliefs differently, in a static Nash equilibrium (see Kyle (1989)) set up of two agents model when agents hold incorrect but strategic beliefs, Jouini and Napp (2009) provide a discipline for belief formation through a model of subjective beliefs in order to provide a rationale for belief heterogeneity. They find that optimism (overconfidence) as well as pessemism (doubt) emerge as optimal beliefs of agents' strategic behaviour and there is a positive correlation between pessimism (doubt) and risk tolerance. This strategic explanation of heterogeneous beliefs is in contrast with rational approach to beliefs where agents try to reflect the 'world as it is' in their beliefs, and with approach in which forward-looking agents optimally distort beliefs and in which beliefs are of intrinsic value to agents, as with wishful thinking or fear of disappointment (see Brunnermeier and Parker (2005)).

By introducing a concept of pragmatic beliefs, Hvide (2002) uses a simple gametheoretic example of a job market and shows optimism can be the equilibrium outcome if agents form beliefs pragmatically. The main justification for programmatic beliefs is dynamics in the sense that, without awareness about their own optimism, agents are gradually learning that a certain way of forming beliefs is more rewarding than other ways. Also, by quantifying the amount of pessimism and doubt in survey data on US consumption and income, Giordani and Soderlind (2006) find some evidence of pessimism, but individual forecasters clearly exhibit overconfidence rather than doubt. By showing that the average distribution shows no statistically significant sign of either overconfidence or doubt, they conclude that doubt is not a promising explanation of the equity premium puzzle and the amount of pessimism provides only a rather small improvement in the empirical performance of the model.

The above literature shows that there appears an inconsistency between the theoretic and empirical (or experimental) results, illustrating the complicated impact of the heterogeneity in beliefs. This indicates a need to examine the complicated impact of the heterogeneity on the market explicitly. To this need, in this paper, we consider a simple financial market with two risky assets, one risk-free asset, and two agents who have different preferences and biased beliefs. Both agents have homogeneous beliefs in the return for one of the risky assets, but have biased beliefs in the return of the other asset. The heterogeneity of agents is characterized by their difference in risk tolerance, the expected returns, the standard deviations and, in particular, the correlation of the two asset returns, which has not been examined in the literature since most of them consider the situation of only one risky asset. The biased beliefs are assumed to be mean preserved spreads of a benchmark homogeneous belief. By assuming that agents maximize a primitive utility function (see Sharpe (1991) and Levy 
and Markowitz (1979)), agents choose their optimal portfolios based on their beliefs. By constructing a consensus belief, the market equilibrium is characterized by the consensus belief. Different from the standard rational expectation equilibrium, the market equilibrium under the consensus belief reflects the bounded rationality of the agents in the sense that the market equilibrium is achieved when agents make their optimal decision based on their subjective beliefs. We call such equilibrium as a boundedly rational equilibrium. We show that the different aspects of the biased beliefs, including the optimism/pessemism and confidence/doubt, can have different impact on the market equilibrium. In particular, the biased beliefs in return correlation have significantly impact on the equilibrium market equity premium and risk-free rate.

The paper is structured as follows. In Section 2, we set up the economy and describe the aggregation problem when agents have heterogeneous preferences and beliefs. We show how the different risk tolerance and heterogeneous beliefs can be aggregated through a consensus belief in market equilibrium. In particular, we derive a CAPM under heterogeneous beliefs. In Section 3, as a benchmark of our analysis, we include the traditional CAPM under the homogeneous belief in terms of market portfolio, the equilibrium risk-free rate and market risk premium and illustrate the equity premium and risk-free rate puzzles. In Section 4, by introducing biased risk preference and biased beliefs among two agents, we examine the impact of the heterogeneity on the market equilibrium risk-free rate and market premium explicitly. In particular, we explore the conditions in explanting the risk-free rate and equity premium puzzles. Finally, some concluding remarks are presented in Section 5.

\section{The Heterogeneous Beliefs and Boundedly Rational Equilibrium}

2.1. The Economy. We consider a two-date economy ${ }^{2}$ in which there are two risky assets, indexed by $j=1,2$ and two agents, indexed by $i=1,2$. Assume agents have different preferences. Asset $j(j=1,2)$ offers investors a end-of-period return of $\tilde{r}_{j}$. The joint probability distribution of the returns of the two assets are assumed to be jointly normal but unknown to the agents. This implies that, based on the same information set, agents have heterogeneous beliefs in the expected returns and variancecovariance of the returns. For agent $i(i=1,2)$, let $\tau_{i}$ be his/her risk tolerance, and

$$
\boldsymbol{\mu}_{i}=\left(\begin{array}{c}
\mu_{i, 1} \\
\mu_{i, 2}
\end{array}\right) \quad \text { and } \quad V_{i}=\left(\begin{array}{cc}
\sigma_{i, 1}^{2} & \rho_{i} \sigma_{i, 1} \sigma_{i, 2} \\
\rho_{i} \sigma_{i, 1} \sigma_{i, 2} & \sigma_{i, 2}^{2}
\end{array}\right)
$$

be his/her beliefs in the means and covariance matrix, respectively, where

$$
\mu_{i, j}=1+\mathbb{E}_{i}\left(\tilde{r}_{j}\right), \quad \sigma_{i, j}^{2}=\operatorname{Var}_{i}\left(\tilde{r}_{j}\right), \quad \rho_{i}=\operatorname{Correl}_{i}\left(\tilde{r}_{1}, \tilde{r}_{2}\right)
$$

\footnotetext{
${ }^{2}$ The discussion and results of this section for the general economy of many risky assets and many heterogeneous beliefs can be found in He and Shi (2009).
} 
for $i, j=1,2$. We use $\mathcal{B}_{i}:=\left(\mu_{i, 1}, \mu_{i, 2}, \sigma_{i, 1}, \sigma_{i, 2}, \rho_{i}\right)$ to denote the beliefs of agent $i$.

2.2. Optimal Portfolio Problem. Agent $i$ 's terminal wealth is $\tilde{W}_{i}=W_{i 0}\left(\boldsymbol{\pi}_{i}^{T} 1+\tilde{r}_{j}\right)$, where $\boldsymbol{\pi}_{i}=\left(\pi_{i, 1}, \pi_{i, 2}\right)^{T}$ is the vector of portfolio weights (proportion of wealth invested in each asset). We assume that investors maximize a primitive utility function $U_{i}(\boldsymbol{\pi})=\boldsymbol{\pi}_{i}^{T} \boldsymbol{\mu}_{i}-\frac{1}{2 \tau_{i}} \boldsymbol{\pi}_{i}^{T} V_{i} \boldsymbol{\pi}_{i}$ under the budget constraint is $\mathbf{1}^{T} \boldsymbol{\pi}_{i}=1$, where $\mathbf{1}=(1,1)^{T}$ and $\tau_{i}$ is the risk-tolerance that measures the marginal rate of substitution of variance for expected return. This utility function has been used in Sharpe (1991), it is consistent with Markowitz portfolio selection criterion and also serves as a good approximation for other type of utility functions, see Levy and Markowitz (1979). Solving this standard portfolio optimization problem leads to the optimal portfolio weights given by

$$
\boldsymbol{\pi}_{i}^{*}=\tau_{i} V_{i}^{-1}\left(\boldsymbol{\mu}_{i}-\lambda_{i}^{*} \mathbf{1}\right), \quad \lambda_{i}^{*}=\frac{\mathbf{1}^{T} V_{i}^{-1} \boldsymbol{\mu}_{i}-1 / \tau_{i}}{\mathbf{1}^{T} V_{i}^{-1} \mathbf{1}},
$$

where $\lambda_{i}^{*}$ measures the marginal certainty equivalent rate of return (CER) per one percent investment in each asset. In the case when there exists a risk-free security with a certain future payoff $R_{f}=1+r_{f}$, the CER of agents becomes $R_{f}$ and the optimal portfolio can be simplified to

$$
\boldsymbol{\pi}_{i}^{*}=\tau_{i} V_{i}^{-1}\left(\boldsymbol{\mu}_{i}-R_{f} \mathbf{1}\right) .
$$

2.3. Consensus Belief and Boundedly Rational Equilibrium. We characterize market equilibrium by the concept of a consensus belief developed in Chiarella, Dieci and $\mathrm{He}$ (2006) with a riskfree asset and He and Shi (2009) without riskfree asset. Essentially, the consensus belief reflects the aggregation of the heterogeneous beliefs when the market is in equilibrium. It helps us to understand how heterogeneity or biases in agents' beliefs can affect the endogenous variables derived from market equilibrium such as the market risk premium, risk-free rate and market volatility.

Definition 2.1. A belief $\mathcal{B}_{a}=\left(\mu_{a, 1}, \mu_{a, 2}, \sigma_{a, 1}, \sigma_{a, 2}, \rho_{a}\right)$ is called a market consensus belief of the two agent economy if the equilibrium price vector of the risky assets (and the risk-free rate when there exists a risk-free asset) under the heterogeneous beliefs $\mathcal{B}_{i}:=\left(\mu_{i, 1}, \mu_{i, 2}, \sigma_{i, 1}, \sigma_{i, 2}, \rho_{i}\right)(i=1,2)$ is also the market equilibrium price vector of the risky assets (and the risk-free rate) under the homogeneous belief $\mathcal{B}_{a}$.

In the following, we show that such consensus belief for the economy with heterogeneous beliefs can be constructed. Let $W_{i 0}$ be the initial wealth of agent $i(i=1,2)$. Then $W_{0}=W_{10}+W_{20}$ corresponds to the total market wealth. Define the market wealth proportion $w_{i}=\frac{W_{i 0}}{W_{0}}$ of agent $i(i=1,2)$, then the market clearing condition

$$
W_{o} \boldsymbol{\pi}_{m}=W_{10} \boldsymbol{\pi}_{1}^{*}+W_{20} \boldsymbol{\pi}_{2}^{*}
$$


becomes

$$
\boldsymbol{\pi}_{1}^{*} w_{1}+\boldsymbol{\pi}_{2}^{*} w_{2}=\boldsymbol{\pi}_{m},
$$

where $\boldsymbol{\pi}_{m}$ denotes the market portfolio of risky assets. Since the market equilibrium is obtained based on the fact that both agents make their optimal portfolio decision under their subjective beliefs, rather than the objective belief, we call such equilibrium as Boundedly Rational Equilibrium (BRE). The following result characterizes such BRE.

Proposition 2.2. Let

$$
\tau_{a}:=w_{1} \tau_{1}+w_{2} \tau_{2}, \quad \lambda_{a}^{*}:=w_{1} \frac{\tau_{1}}{\tau_{a}} \lambda_{1}^{*}+w_{2} \frac{\tau_{2}}{\tau_{a}} \lambda_{2}^{*} .
$$

Then

(i) the consensus belief $\mathcal{B}_{a}$ is given by

$$
\begin{gathered}
V_{a}^{-1}=\frac{1}{\tau_{a} \lambda_{a}^{*}}\left[w_{1} \tau_{1} \lambda_{1}^{*} V_{1}^{-1}+w_{2} \tau_{2} \lambda_{2}^{*} V_{2}^{-1}\right] \\
\boldsymbol{\mu}_{a}=\mathbb{E}_{a}(\mathbf{1}+\mathbf{r})=\frac{1}{\tau_{a}}\left[w_{1} \tau_{1}\left(V_{1}^{-1} V_{a}\right) \boldsymbol{\mu}_{1}+w_{2} \tau_{2}\left(V_{2}^{-1} V_{a}\right) \boldsymbol{\mu}_{2}\right]
\end{gathered}
$$

(ii) the equilibrium market portfolio is determined by

$$
\boldsymbol{\pi}_{m}=\tau_{a} V_{a}^{-1}\left(\boldsymbol{\mu}_{a}-\lambda_{a}^{*} \mathbf{1}\right)
$$

(iii) the Zero-beta CAPM relation

$$
\mathbb{E}_{a}[\tilde{\mathbf{r}}]-\left(\lambda_{a}^{*}-1\right) \mathbf{1}=\boldsymbol{\beta}\left[\mathbb{E}_{a}\left(\tilde{r}_{m}\right)-\left(\lambda_{a}-1\right)\right],
$$

holds under the consensus belief $\mathcal{B}_{a}$, where

$$
\boldsymbol{\beta}=\left(\beta_{1}, \beta_{2}\right)^{T}, \quad \beta_{j}=\frac{\operatorname{Cov}_{a}\left(\tilde{r}_{m}, \tilde{r}_{j}\right)}{\sigma_{a}^{2}\left(\tilde{r}_{m}\right)}, \quad j=1,2 .
$$

Proposition 2.2 can be proved similarly to the proof of Proposition 5.1 in He and Shi (2009). Based on Proposition 2.2, we have the following observations. (i) The risk tolerance of the market is a weighted average of that of the two agents weighted by the corresponding wealth fraction of the agents. Note that the wealth weighted risk tolerance $w_{i} \tau_{i}(i=1,2)$ also appears in the consensus beliefs defined in (2.3) and (2.4). Therefore, if we can treat $w_{i} \tau_{i}(i=1,2)$ as a risk tolerance, this implies the assumption that wealthier investors are more risk tolerant. (ii) The consensus belief of the variance/covariance matrix can be treated as a harmonic mean of agents' subjective beliefs weighted by their wealth weighted risk-tolerance and CER. Hence a more risktolerant investor with a higher CER will dominate the consensus belief and hence the market. (iii) The consensus belief of expected asset returns is also an average of investors' subjective beliefs weighted by their wealth weighted risk-tolerance and the inverse of their covariance matrices. 
In general, the impact of different risk tolerance and heterogeneous beliefs of agents can have a very complicated impact, in particular, the marginal certainty equivalent rate of return $\lambda_{i}^{*}$ for investor $i$ can be different. However, if there exist a risk-free security, then $\lambda_{i}^{*}=R_{f}$ for all $i$ in equations (2.3) and (2.4). In this case, by assuming that the risk-free asset is in zero-net supply in market equilibrium, we obtain the following Corollary.

Corollary 2.3. If there exist a risk-free security with return of $R_{f}=1+r_{f}$, then the consensus belief $\mathcal{B}_{a}$ is given by

$$
\begin{gathered}
V_{a}^{-1}=\frac{1}{\tau_{a}}\left[w_{1} \tau_{1} V_{1}^{-1}+w_{2} \tau_{2} V_{2}^{-1}\right] \\
\boldsymbol{\mu}_{a}=\mathbb{E}_{a}(\mathbf{1}+\tilde{\mathbf{r}})=\frac{1}{\tau_{a}}\left[w_{1} \tau_{1}\left(V_{1}^{-1} V_{a}\right) \boldsymbol{\mu}_{1}+w_{2} \tau_{2}\left(V_{2}^{-1} V_{a}\right) \boldsymbol{\mu}_{2}\right]
\end{gathered}
$$

the market portfolio becomes

$$
\boldsymbol{\pi}_{m}=\tau_{a} V_{a}^{-1}\left(\mathbb{E}_{a}(\tilde{\mathbf{r}})-r_{f} \mathbf{1}\right)
$$

the equilibrium CAPM relation becomes

$$
\mathbb{E}_{a}[\tilde{\mathbf{r}}]-r_{f} \mathbf{1}=\boldsymbol{\beta}\left[\mathbb{E}_{a}\left(\tilde{r}_{m}\right)-r_{f}\right]
$$

and the equilibrium risk-free rate is given by

$$
r_{f}=\frac{\mathbf{1}^{T} V_{a}^{-1} \mathbb{E}_{a}(\tilde{\mathbf{r}})-\frac{1}{\tau_{a}}}{\mathbf{1}^{T} V_{a}^{-1} \mathbf{1}} .
$$

A similar result to Corollary 2.3 for many assets and beliefs can be found in Chiarella, Dieci and $\mathrm{He}$ (2009) and $\mathrm{He}$ and Shi (2009). The impact of the heterogeneity on the market equilibrium, CAPM relation, market equity premium, and risk free rate can be complicated in general. By focusing on the case of two assets and two agents in this paper, we are able to examine explicitly the impact in Section 4. To compare with the traditional CAPM, we first consider the homogeneous belief as the benchmark case in the next section.

\section{A BenCHMARK CASE UNDER homogeneous BelieF}

To examine the impact of the heterogeneity on the market equilibrium and compare with the market equilibrium under a homogeneous belief, we consider in this section a benchmark case under the standard rational expectation in which both agents may have different risk tolerance, but have the same beliefs in returns. ${ }^{3}$, denoted by $\mathcal{B}_{o}=$ $\left(\mu_{1}, \mu_{2}, \sigma_{1}, \sigma_{2}, \rho\right)$, that is $\mathcal{B}_{i}=\mathcal{B}_{o}$ for $i=1,2$. In this benchmark case, we have from

${ }^{3}$ The benchmark beliefs $\mathcal{B}_{o}$ can be treated as either the objective belief or a benchmark homogeneous belief. 
Proposition 2.2 that

$$
V_{a}=\left(\begin{array}{cc}
\sigma_{1}^{2} & \rho \sigma_{1} \sigma_{2} \\
\rho \sigma_{1} \sigma_{2} & \sigma_{2}^{2}
\end{array}\right):=V_{o}, \quad \boldsymbol{\mu}_{a}=\left(\begin{array}{c}
\mu_{1} \\
\mu_{2}
\end{array}\right):=\boldsymbol{\mu}_{o} .
$$

Consequently, the market portfolio is simply given by

$$
\hat{\boldsymbol{\pi}}_{m}:=\frac{1}{\sigma_{1}^{2}+\sigma_{2}^{2}-2 \rho \sigma_{1} \sigma_{2}}\left(\begin{array}{c}
\tau_{a}\left(\mu_{1}-\mu_{2}\right)+\sigma_{2}\left(\sigma_{2}-\rho \sigma_{1}\right) \\
\tau_{a}\left(\mu_{2}-\mu_{1}\right)+\sigma_{1}\left(\sigma_{1}-\rho \sigma_{2}\right.
\end{array}\right) .
$$

When there exists a risk-free asset, the market risk-premium, risk-free return and market variance are given, respectively, by

$$
\begin{aligned}
& \hat{\mathbb{E}}\left(\tilde{r}_{m}-r_{f}\right):=\frac{\left(\mu_{1}-\mu_{2}\right)^{2} \tau_{a}^{2}+\left(1-\rho^{2}\right) \sigma_{1}^{2} \sigma_{2}^{2}}{\tau_{a}\left(\sigma_{1}^{2}+\sigma_{2}^{2}-2 \rho \sigma_{1} \sigma_{2}\right)} \\
& \hat{R}_{f}:=\frac{\sigma_{1}^{2} \sigma_{2}^{2}}{\sigma_{1}^{2}+\sigma_{2}^{2}-2 \rho \sigma_{1} \sigma_{2}}\left(\frac{\mu_{1}}{\sigma_{1}^{2}}+\frac{\mu_{2}}{\sigma_{2}^{2}}-\frac{\rho\left(\mu_{1}+\mu_{2}\right)}{\sigma_{1} \sigma_{2}}-\frac{1}{\tau_{a}}\left(1-\rho^{2}\right)\right), \\
& \hat{\sigma}^{2}\left(\tilde{r}_{m}\right):=\frac{\left(\mu_{1}-\mu_{2}\right)^{2} \tau_{a}^{2}+\left(1-\rho^{2}\right) \sigma_{1}^{2} \sigma_{2}^{2}}{\left(\sigma_{1}^{2}+\sigma_{2}^{2}-2 \rho \sigma_{1} \sigma_{2}\right)}
\end{aligned}
$$

It is easy to see that $\tau_{a}=\hat{\sigma}^{2}\left(\tilde{r}_{m}\right) / \hat{\mathbb{E}}\left(\tilde{r}_{m}-r_{f}\right)$, so the market risk-tolerance represents the marginal rate of substitution between market risk premium and market variance.

Weil (1989) attributes the risk premium puzzle and the risk-free rate puzzle to two distinction basic empirical facts about the aggregate consumption process. Essentially, under the representative agent framework, on the one hand, there is not enough individual consumption risk, so if agents are only moderately risk-averse, the observed risk premium is too high. On the other hand, the average rate of growth of individual consumption is too high and to explain the observed low risk-free rate, agents need to be extremely averse to intertemporal substitution. Within the simple economy considered in this paper, we show that similar puzzles can still arise in the homogeneous benchmark case when asset returns are correlated and the asset with a higher expected future return is also much more risky. To illustrate this, we consider the following numerical example.

Example 3.1. Let the two risky assets in the economy have expected returns $\left(\mu_{1}, \mu_{2}\right)=$ $(1.06,1.09)$ and standard deviations $\left(\sigma_{1}, \sigma_{2}\right)=(0.08,0.3)$ and correlation coefficient $\rho=0.8$. Both investors hold the benchmark belief, that is, $\mathcal{B}_{i}=\mathcal{B}_{o}=\left(\mu_{1}, \mu_{2}, \sigma_{1}, \sigma_{2}, \rho\right)$. For simplicity, we also assume that each investor has half of the aggregate market initial wealth (so that $\left.w_{1}=w_{2}=1 / 2\right)^{4}$.

We choose $\tau_{i}=0.5(i=1,2)$, which is a reasonable level of risk-tolerance. Note that $\tau_{a}=\hat{\sigma}^{2}\left(\tilde{r}_{m}\right) / \hat{\mathbb{E}}\left(\tilde{r}_{m}-r_{f}\right)$, this implies that the market in equilibrium requires $2 \%$

\footnotetext{
${ }^{4}$ Alternatively, we can treat $w_{i} \tau_{i}$ as the risk tolerance of agent $i$ and argue that wealthier agents are more risk tolerant. Therefore, in the rest of the paper, we also assume that $w_{1}=w_{2}=1 / 2$.
} 
expected excess return (above the risk-free rate) for $10 \%$ standard deviation. Consequently, we have from equations (3.1) and (3.2) that the market portfolio is given by $\boldsymbol{\pi}_{m}=(0.962,0.038)^{T}$ and

$$
\tau_{a}=0.5, \quad \hat{r}_{f}=4.62 \%, \quad \hat{\mathbb{E}}\left(\tilde{r}_{m}-r_{f}\right)=1.49 \%, \quad \text { and } \quad \hat{\sigma}_{m}=8.63 \% .
$$

Clearly with $\tau_{i}=\tau_{a}=0.5$, the risk-free rate is too high, the risk-premium and market volatility are too low. We can certainly increase the risk-premium and reduce the risk-free rate by decreasing $\tau_{a}$, however this leads to some absurd implications. For example, we could choose $\tau_{a}=0.1$, then

$$
\hat{r}_{f}=0 \%, \quad \hat{\mathbb{E}}\left(\tilde{r}_{m}-r_{f}\right)=6 \%, \quad \text { and } \quad \hat{\sigma}_{m}=8 \% .
$$

In this case, the market portfolio becomes $\boldsymbol{\pi}_{m}=(1,0)^{T}$ (asset 2 is redundant), also the market requires $10 \%$ expected excess return for $10 \%$ standard deviation which does not seem reasonable. This simple example illustrates the equity premium and risk-free puzzles under the homogeneous belief in the literature.

\section{THE IMPACT OF Heterogeneity}

In order to explore the impact of heterogeneity in investors' belief on the market equilibrium, in particular, the risk-premium and the risk-free rate, we assume that there is a risk-free asset in most of our analysis in this section. However, the case when there is no risk-free asset will be examined later. To examine the impact explicitly, we assume that agents agree about the first risky asset (which might be well informed) but disagree about the expected return, standard deviation of the second asset (which is less informed) and the correlation coefficient of the returns of the two assets. The disagreements are characterized by mean-preserved spread about the benchmark belief. That is, the beliefs in the expected return and the standard deviation of the first asset for both agents are given by the benchmark beliefs: $\left(\sigma_{i, 1}, \mu_{i, 1}\right)=\left(\sigma_{1}, \mu_{1}\right)$ for $i=1,2$, while the risk tolerance and the beliefs of the two agents in the expected return and standard deviation of the second asset, and the return correlation of the two assets are mean-preserved spread of the benchmark belief. More precisely, we assume that the risk-tolerances of the two agents are given, respectively, by

$$
\tau_{1}=\tau_{o}(1-\Delta), \quad \tau_{2}=\tau_{o}(1+\Delta), \quad \Delta \in(-1,1) ;
$$

the beliefs about the standard deviation of asset 2 are given by

$$
\sigma_{1,2}=\sigma_{2}(1-\delta), \quad \sigma_{2,2}=\sigma_{2}(1+\delta), \quad \delta \in(-1,1) ;
$$

the beliefs about the correlation between asset returns are given by

$$
\rho_{1}=\rho(1-\varepsilon), \quad \rho_{2}=\rho(1+\varepsilon), \quad \varepsilon \in(-1,1) ;
$$


and the beliefs of expected returns of asset 2 are given by

$$
\mu_{1,2}=\mu_{2}(1-\alpha), \quad \mu_{2,2}=\mu_{2}(1+\alpha), \quad \alpha \in(-1,1) .
$$

The mean-preserved spreads imply that the average risk-tolerance and belief in this heterogeneous economy is exactly the same as the benchmark homogeneous economy. However, the consensus belief may not be same as the benchmark belief. As the result, the market portfolio, market risk-premium, risk-free rate and the market volatility may also differ from the homogeneous benchmark economy. For this setup, the different aspects of the heterogeneity are characterized by $\Delta, \delta, \varepsilon$ and $\alpha$. To examine the joint impact of risk tolerance, optimism/pessism, and confidence/doubt on the market, we consider four combinations of these parameters in the following.

4.1. Case 1: Impact of Risk Tolerance and Optimism/Pessimism. We first consider the case where the two agents have different risk-tolerance and heterogeneous belief regarding the expected future return of asset 2 , that is

$$
\delta=0, \quad \varepsilon=0, \quad \Delta \in(-1,1), \quad \alpha \in(-1,1) .
$$

This means that agent1 is less (more) risk tolerance than agent 2 when $\Delta>0(<$ 0 ) and agent 1 is more pessimistic (optimistic) than agent 2 when $\alpha>0(<0)$. In particular, when $\Delta \alpha>0(<0)$, the risk tolerance and optimism of the two agents are positively (negatively) correlated, meaning that the more risk-tolerant investor is optimistic, while the less risk-tolerant investor is pessimistic, about future asset return. Applying Corollary 2.3 to this case, we obtain the following result.

Corollary 4.1. For the case (4.1), the consensus belief is given by

$$
\tau_{a}=\tau_{o}, \quad V_{a}=V_{o}, \quad \boldsymbol{\mu}_{a}=\left(\mu_{1}, \mu_{2}(1+\alpha \Delta)\right)^{T} .
$$

Consequently,

(i) the change in market portfolio is given by

$$
\boldsymbol{\pi}_{m}-\hat{\boldsymbol{\pi}}_{m}=\frac{\alpha \Delta \tau_{o} \mu_{2}}{\sigma_{1}^{2}-2 \rho \sigma_{1} \sigma_{2}+\sigma_{2}^{2}}\left(\begin{array}{l}
1 \\
1
\end{array}\right)
$$

(ii) the change in risk-premium is given by

$$
\left(\mathbb{E}\left(\tilde{r}_{m}\right)-r_{f}\right)-\left(\hat{\mathbb{E}}\left(\tilde{r}_{m}\right)-\hat{r}_{f}\right)=\alpha \Delta \mu_{2} \frac{\sigma_{1}\left(\rho \sigma_{2}-\sigma_{1}\right)+\tau_{o}\left(\mu_{2}-\mu_{1}\right)}{\sigma_{1}^{2}-2 \rho \sigma_{1} \sigma_{2}+\sigma_{2}^{2}} ;
$$

(iii) the change in risk-free rate is given by

$$
\hat{r}_{f}-r_{f}=\alpha \Delta \sigma_{1} \mu_{2} \frac{\rho \sigma_{2}-\sigma_{1}}{\sigma_{1}^{2}-2 \rho \sigma_{1} \sigma_{2}+\sigma_{2}^{2}}
$$


(iv) the change in market volatility is given by

$$
\sigma_{m}^{2}-\hat{\sigma}_{m}^{2}=\alpha \Delta \tau_{o}^{2} \mu_{2} \frac{\left(\mu_{2}-\mu_{1}\right)+\left(\mu_{2}(1+\alpha \Delta)-\mu_{1}\right)}{\sigma_{1}^{2}-2 \rho \sigma_{1} \sigma_{2}+\sigma_{2}^{2}} ;
$$

(v) the changes in the beta coefficients are given by

$$
\begin{aligned}
& \beta_{1}=\hat{\beta}_{1}+\alpha \Delta \mu_{2} \tau_{o} \frac{\rho \sigma_{2}-\sigma_{1}}{\sigma_{1}^{2}-2 \rho \sigma_{1} \sigma_{2}+\sigma_{2}^{2}}, \\
& \beta_{2}=\hat{\beta}_{2}+\alpha \Delta \mu_{2} \tau_{o} \frac{\sigma_{2}-\rho \sigma_{1}}{\sigma_{1}^{2}-2 \rho \sigma_{1} \sigma_{2}+\sigma_{2}^{2}} .
\end{aligned}
$$

where $\left(\hat{\beta}_{1}, \hat{\beta}_{2}\right)^{T}=V_{o} \hat{\boldsymbol{\pi}}_{m} / \hat{\sigma}_{m}^{2}$ are the asset betas under the homogeneous benchmark case.

Corollary 4.1 characterize explicitly the impact of the heterogeneity on the market. It is easy to see that if either both the agents have the same risk preference (so that $\Delta=0$ ) or they have the same benchmark belief in the expected return of both assets (so that $\alpha=0$ ), then $\alpha \Delta=0$ and the results for the heterogeneous beliefs are reduced to that for the benchmark homogeneous case. The impact of the heterogeneity in this case (4.1) depends on the sign of $\alpha \Delta$ and the return correlation $\rho$ in the benchmark belief. Corollary 4.1 leads to following three implications.

Firstly, when risk-tolerance and optimism about future returns are positively (negatively) correlated, that is $\alpha \Delta>(<) 0$, it follows from (4.2) that the aggregate market is optimistic (pessimistic) about the expected return of the second asset. Consequently, the aggregate market, indicated by the market portfolio in (4.3), invests more (less) into asset 2 and less (more) into asset 1 and that the market volatility measured by $\sigma_{m}$ is high (low) following (4.5). This observation that the market becomes pessimistic when the risk tolerance and pessimism are positively correlated is also found in Jouini and Napp (2006).

Secondly, comparing with the benchmark belief case, we have from (4.4) that the market with biased beliefs among the two agents increase the market risk-premium when either

$$
\alpha \Delta>0 \quad \text { and } \quad \mu_{2}-\mu_{1}>\sigma_{1}\left(\sigma_{1}-\rho \sigma_{2}\right) / \tau_{o}
$$

or

$$
\alpha \Delta<0 \quad \text { and } \quad \mu_{2}-\mu_{1}<\sigma_{1}\left(\sigma_{1}-\rho \sigma_{2}\right) / \tau_{o} .
$$

Similarly, from (4.5), the risk-free rate under the biased belief is reduced when either

$$
\alpha \Delta>0 \quad \text { and } \quad \rho>\sigma_{1} / \sigma_{2}
$$

or

$$
\alpha \Delta<0 \quad \text { and } \quad \rho<\sigma_{1} / \sigma_{2} .
$$

In other words, when either (i) the risk tolerance and optimism of agent are positively correlated, returns of the two assets are highly positively correlated (so that $\rho>$ 
$\left.\sigma_{1} / \sigma_{2}\right)$, and also the difference in asset expected returns are large enough $\left(\mu_{2}-\mu_{1}>\right.$ $\sigma_{1}\left(\sigma_{1}-\rho \sigma_{2}\right) / \tau_{o}$ ), or (ii) the risk tolerance and optimism of agent are negatively correlated, returns of the two assets are less (even negatively) correlated (so that $\rho<\sigma_{1} / \sigma_{2}$ ), and difference in asset expected returns are small enough $\left(\mu_{2}-\mu_{1}<\sigma_{1}\left(\sigma_{1}-\rho \sigma_{2}\right) / \tau_{o}\right)$, then the biased beliefs increase the market premium and reduce the risk-free rate. This observation helps us to resolve the equity premium and risk-free rate puzzles. Within the framework of heterogeneous beliefs, some literature (see, for example, Abel (2002) and Jouini and Napp (2006)) explain the puzzles by arguing that it is sufficient when the risk tolerance and pessimism are positively correlated. Our analysis shows that both positive and negative correlations between the risk tolerance and pessimism can explain the puzzles, depending on the return correlation. To our knowledge, the role of the return correlation on the explanation of the puzzles has not been explored in the literature and we will show that it plays a very important role towards the puzzles.

Thirdly, the standard CAPM relation under the benchmark belief is no longer held, though the CAPM under the heterogeneous beliefs still holds under the consensus belief. It is easy to see that, once the biases in the expected return disappear, the betas become the standard betas under the benchmark belief. Under the biased beliefs, the betas $\beta_{j}$ can be decomposed into the betas $\hat{\beta}_{j}$ under the benchmark belief and a term related to the biases in the beliefs, which becomes a risk factor under the heterogeneous CAPM relation. As part of the $\beta_{j}$, this risk factor related to the biased beliefs becomes part of the systematic risk which is missing in the standard CAPM relation. However, this risk factor can be either positive or negative for different asset. Comparing with the benchmark case, $\beta_{1}>\hat{\beta}_{1}$ under either condition (4.10) or condition (4.11), in other words, $\beta_{1}$ changes in the same direction as the risk-free rate. However, the change of systematic risk for asset 2 has no clear relation with the change in risk-free rate, the sign of $\beta_{2}>\hat{\beta}_{2}$ depends on the terms $\alpha \Delta$ and $\sigma_{2}-\rho \sigma_{1}$. For example, if asset 2 is more risky and $\alpha \Delta<0$, then heterogeneity can actually reduce the systematic risk of the second asset.

To assess the exact impact, we now conduct a numerical analysis. Based on the numerical values provided in Example 3.1, we show graphically in Figure 4.1 the impact of heterogeneity in terms of $\alpha$ and $\Delta$ on the change of market portfolio (in terms of the second risky asset in the market portfolio) in Figure 4.1(a), the market volatility in Figure 4.1(b), the expected market return in Figure 4.1(c), and the equilibrium riskfree rate in market equilibrium in Figure 4.1(d). Note that we have $\rho>\sigma_{1} / \sigma_{2}$ for the numerical values. It is clear that the plots are symmetric reflecting the fact the effect of heterogeneity depends on the product $\alpha \Delta$ rather than individually. We see in this case that as the product $\alpha \Delta$ gets larger, the market portfolio consists more of asset 2, which leads to higher market return and volatility, at the same time the risk-free rate 


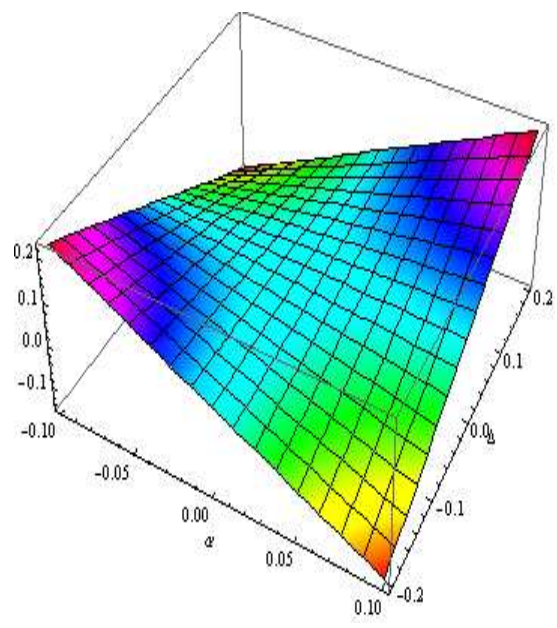

(a1) Market Proportion of asset 2

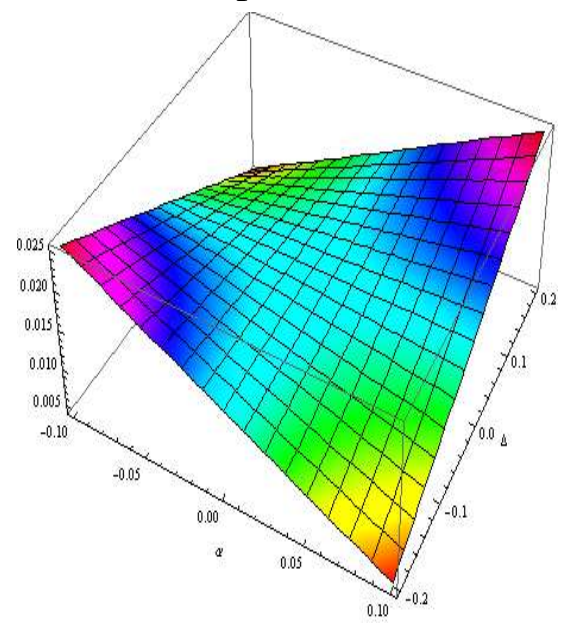

(a3) Risk premium

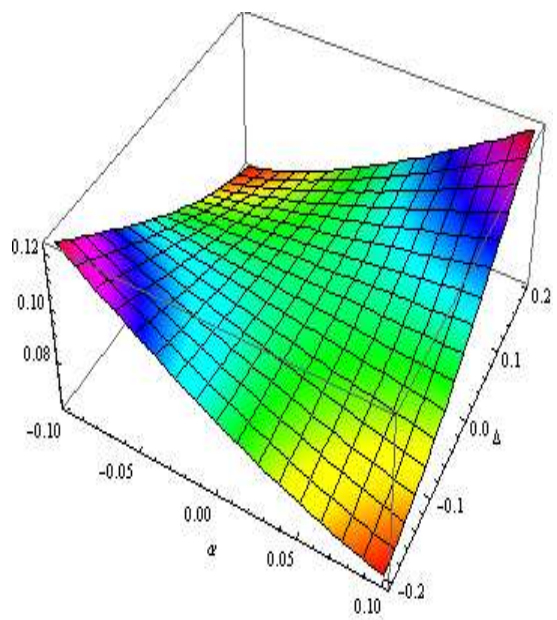

(a2) Market volatility

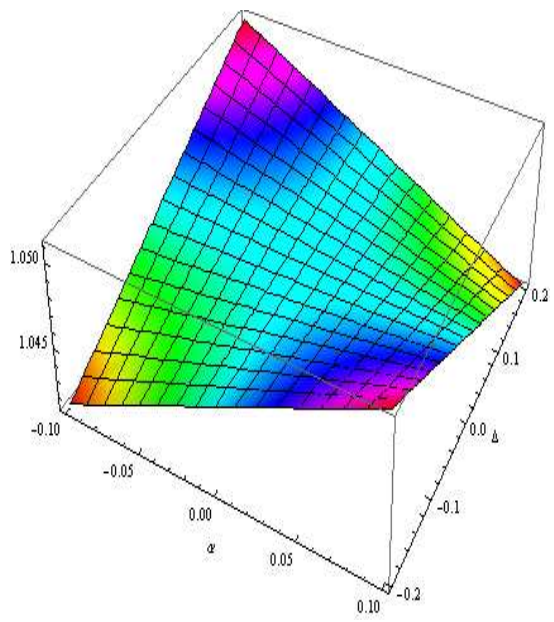

(a4) Risk-free rate

FIGURE 4.1. Effect of heterogeneity in risk-tolerance $\Delta$ and beliefs of expected return $\alpha$ on the market proportion of asset 2, market volatility, market risk-premium and the risk-free rate

reduces and the risk-premium increases. Also, the Sharpe ratio of the market portfolio increases, which suggests that heterogeneity of $\alpha \Delta$ improves the mean-variance efficiency of the aggregate market.

To quantify the impact on the market, we base on the numerical values provided in Example 3.1 and choose $(\Delta, \delta, \varepsilon, \alpha)=(0.2,0,0,0.1)$. The results are reported in Table 4.1. Comparing with the benchmark homogeneous belief, the results for Case 1 in Table 4.1 show that heterogeneity in the risk tolerance and the expected return helps to resolve the puzzles when $\alpha \Delta>0$. However, the overall effect is not significant for the chosen parameters. Risk premium increases moderately by $1 \%$ and the riskfree rate is merely reduced by less than half of a percent. This is mainly because that 


\begin{tabular}{|c|c|c|c|c|c|c|}
\hline Cases & $(\Delta, \delta, \varepsilon, \alpha)$ & $\pi_{m, 2}$ & $\sigma\left(\tilde{r}_{m}\right)$ & $\mathbb{E}\left(\tilde{r}_{m}-r_{f}\right)$ & $r_{f}$ & $\frac{\mathbb{E}\left(\tilde{r}_{m}\right)-r_{f}}{\sigma_{m}}$ \\
\hline Benchmark & $(0,0,0,0)$ & 0.038 & $8.63 \%$ & $1.49 \%$ & $4.62 \%$ & 0.1727 \\
\hline Case 1 & $(0.2,0,0,0.1)$ & 0.2258 & $12.3 \%$ & $2.54 \%$ & $4.14 \%$ & 0.2061 \\
\hline Case 2 & $(0,-0.2,0,0.1)$ & 0.7511 & $24.15 \%$ & $3.88 \%$ & $4.37 \%$ & 0.1606 \\
\hline Case 3 & $(0,0,0.2,0.1)$ & 0.5415 & $19.31 \%$ & $5.69 \%$ & $1.94 \%$ & 0.2947 \\
\hline Case 4 & $(-0.2,0.2,0,0.1)$ & 0.1124 & $10.00 \%$ & $1.77 \%$ & $4.57 \%$ & 0.1770 \\
\hline
\end{tabular}

TABLE 4.1. Effects of heterogeneity on the market proportion of asset $2\left(\pi_{m, 2}\right)$, market volatility $\left(\sigma\left(\tilde{r}_{m}\right)\right)$, market risk-premium $\left(\mathbb{E}\left(\tilde{r}_{m}-r_{f}\right)\right)$, the risk-free rate $\left(r_{f}\right)$ and the Sharpe ratio $\frac{\mathbb{E}\left(\tilde{r}_{m}\right)-r_{f}}{\sigma_{m}}$ for the three cases, compared with the benchmark homogeneous case. Numerical values for the benchmark belief and risk tolerance are given in Example 3.1.

the market becomes over-optimistic which offsets the increase in aggregate volatility, although the market consists more of the riskier asset.

4.2. Case 2: The Impact of Optimism/Pessimism and Confidence/Doubt. In the second case, we focus on the impact of the optimism/pessimism (measured by $\alpha$ ) and confidence/dobut (measured by $\delta$ ) for asset 2 on the market in equilibrium by letting $\Delta=0, \varepsilon=0$. Measured by the beliefs in the standard deviation, agent 1 becomes confident (doubt) when $\delta>0$. Applying Corollary 2.3 to this case, we obtain the following result.

Corollary 4.2. For the second case when $\Delta=0, \varepsilon=0$ and $\delta, \alpha \in(-1,1)$, the consensus belief $\mathcal{B}_{a}=\left(\mu_{a, 1}, \mu_{a, 2}, \sigma_{a, 1}, \sigma_{a, 2}, \rho_{a}\right)$ is given by $\tau_{a}=\tau_{o}$,

$$
\mu_{a, 1}=\mu_{1}-\alpha \delta \mu_{2} \frac{\rho \sigma_{1}}{\sigma_{2}\left(1+\delta^{2}-\rho^{2}\right)}, \quad \mu_{a, 2}=\mu_{2}\left(1-\frac{\alpha \delta\left(2-\rho^{2}\right)}{1-\rho^{2}+\delta^{2}}\right)
$$

and

$$
\begin{aligned}
\sigma_{a, 1}^{2} & =\sigma_{1}^{2}\left[1-\frac{\delta^{2} \rho^{2}}{1+\delta^{2}-\rho^{2}}\right], \quad \sigma_{a, 2}^{2}=\sigma_{2}^{2} \frac{\left(1-\delta^{2}\right)^{2}\left(1-\rho^{2}\right)}{1+\delta^{2}-\rho^{2}} \\
\rho_{a} & =\rho\left[1-\frac{\rho^{2} \delta^{2}}{1+\delta^{2}-\rho^{2}}\right] \frac{\sigma_{1} \sigma_{2}}{\sigma_{a, 1} \sigma_{a, 2}} .
\end{aligned}
$$

Corollary 4.2 gives the explicit impact of the biased beliefs in the expected return and the standard deviation for the second asset among the two agents. One special case is very interesting. This is when there is unbiased beliefs in the standard deviation of the second asset (that is $\delta=0$ ). In this case, we see from (4.12) and (4.13) that there is no difference between the heterogeneous case with biased expected return on the second asset and the benchmark unbiased case, so a biased beliefs in the expected return of the asset 2 alone has no impact on the market. In general, based on (4.12) and (4.13), we see that the biased beliefs in the expected returns of the asset 2 has impact on the market expected return, but not the standard deviations and correlation. 
However, the biased beliefs on the standard deviation of the return of the asset 2 affects the expected returns, standard deviation, and correlation of both assets when the asset returns are correlated. This effect vanishes when $\rho=0$. Corollary 4.2 reflects a joint impact of the optimism/pessimism and confidence/dobut on the market. From equations (4.13), one can see that the aggregate market becomes over-confident when investors have biased beliefs regarding the variance of asset 2's return so that, for $0<\delta<1, \sigma_{a, 1}<\sigma_{1}, \sigma_{a, 2}<\sigma_{2}$ and $\rho_{a} \sigma_{a, 1} \sigma_{a, 2}<\rho \sigma_{1} \sigma_{2}$. From (4.12), when $\alpha \delta<0$, that is when the optimistic (pessimistic) investor is confident (doubtful) about his/her belief of the expected return of asset 2, the market perceives a higher expected return for both assets.

To examine the impact on the market, we let $\delta=-0.2$ and $\alpha=0.1$. This means that the first (second) investor is optimistic (pessimistic) and confident (doubt) on the expected return of the second asset, so that $\alpha \delta<0$. In this case, the results in Table 4.1 show a dramatic increase in the market's holding of asset 2 , since it becomes the riskier asset with a higher expected return. Therefore the market gains in risk-premium but also becomes much more volatile. This is due to the fact that the increase in expected return is much higher for asset 2 than for asset 1 , since based on the numerics in Example 3.1, the value of $\left(\rho \sigma_{1} / \sigma_{2}\right)$ is small relative to $\left(2-\rho^{2}\right)$, see (4.12). The risk-free rate reduces only slightly, because the market consists much more of the riskier asset, but this is offset by the fact that market also becomes over-confident and over-optimistic. This observation is consistent with the survey result in Giordani and Soderlind (2006) that the doubt is not promising explanation of the equity premium puzzle and the amount of pessimism provides only a rather small improvement. The Sharpe ratio drops comparing the homogeneous benchmark case, suggesting that the gain in risk premium cannot compensate for the higher volatility. Although not shown here, a similar plot to Figure 4.1 can be done for this case, suggesting that the effect on equilibrium quantities depend on the joint product $\alpha \delta$.

\subsection{Case 3: The Impact of Optimism/Pessimism and Biased Belief in the Corre-}

lation. In the third case, we examine the joint impact of heterogeneity in the expected return of asset 2 and the correlation coefficient by letting $\Delta=0, \delta=0$ and considering the effect of $(\varepsilon, \alpha)$. When $\epsilon>0(<0)$, agent 1 believes that the return correlation is lower (higher) while agent 2 believes that the return correlation is higher (lower). Applying Corollary 2.3 to this case, we obtain the following result.

Corollary 4.3. For the case that $\Delta=0, \delta=0$ and $\varepsilon, \alpha \in(-1,1)$, the consensus belief $\mathcal{B}_{a}=\left(\mu_{a, 1}, \mu_{a, 2}, \sigma_{a, 1}, \sigma_{a, 2}, \rho_{a}\right)$ is given by $\tau_{a}=\tau_{o}$,

$$
\mu_{a, 1}=\mu_{1}-\alpha \varepsilon \frac{\rho \sigma_{1}}{\left(1-\rho^{2}\right) \sigma^{2}} \mu_{2}, \quad \mu_{a, 2}=\mu_{2}\left[1+\alpha \varepsilon \frac{\rho^{2}}{1-\rho^{2}}\right]
$$




$$
\sigma_{a, 1}^{2}=\sigma_{1}^{2}\left[1-\frac{\varepsilon^{2} \rho^{2}}{1-\rho^{2}}\right], \quad \sigma_{a, 2}^{2}=\sigma_{2}^{2}\left[1-\frac{\varepsilon^{2} \rho^{2}}{1-\rho^{2}}\right], \quad \frac{\rho_{a} \sigma_{a, 1} \sigma_{a, 2}}{\rho \sigma_{1} \sigma_{2}}=1+\frac{\varepsilon^{2} \rho^{2}}{1-\rho^{2}} .
$$

Corollary 4.3 shows the impact of the optimism/pessimism and the biased beliefs in the correlation on the market. Note that the biased beliefs in the expected return of the asset 2 affect the market expected returns of both assets, not the variances and covariance. However, the biased beliefs in the return correlation affect both the first and second moments of the market returns of both assets as well as the return correlation. It is easy to see that, for $0<\varepsilon<1$, we have $\sigma_{a, 1}<\sigma_{1}, \sigma_{a, 2}<\sigma_{2}$ and $\rho_{a} \sigma_{a, 1} \sigma_{a, 2}>\rho \sigma_{1} \sigma_{2}$. This indicates that in aggregate the market becomes more confident about the future returns of the both assets but perceives a higher return covariance comparing to the benchmark case. For $\alpha \varepsilon>0$, that is when the optimistic investor also believes in higher correlation between asset returns, we see from equation (4.14) that the market perceives a higher (lower) expected return for asset 2 (asset 1) when $\rho>0$ and vice versa when $\rho<0$. Intuitively, when $\rho>0$, this should lead the market to invest more into asset 2 . As the result, the aggregate market return and volatility increase. However, different from the previous cases, there is less diversification effect, according to (3.2), hence one should expect a significant reduction in the risk-free rate.

To examine the impact of the heterogeneity in the expected return and correlation, we choose $\epsilon=0.2$ and $\alpha=0.1$ so that $\alpha \varepsilon>0$. The results are given for Case 3 in Table 4.1, showing the most desirable result to resolve the puzzles. The risk-free rate in this case is reduced significantly by nearly $3 \%$ while the risk premium increased significantly by more than $4 \%$. Most noticeably, the Sharpe ratio in this case is 0.2497 , the highest amongst all cases including the homogeneous benchmark by far, implying that the aggregate market becomes the most mean-variance efficient when $\alpha \varepsilon>0$.

4.4. Case 4: The Impact of Risk-tolerance and Confidence/Doubt. In the fouth case, we examine the joint impact of heterogeneity in the risk-tolerance (measure by $\Delta$ ) and confident/doubt (measure by $\delta$ by letting $\alpha=0$ and $\varepsilon=0$. Applying Corollary 2.3 to this case, we obtain the following result.

Corollary 4.4. For the case that $\alpha=0, \varepsilon=0$ and $\Delta, \delta \in(-1,1)$, the consensus belief $\mathcal{B}_{a}=\left(\mu_{a, 1}, \mu_{a, 2}, \sigma_{a, 1}, \sigma_{a, 2}, \rho_{a}\right)$ is given by $\tau_{a}=\tau_{o}, \boldsymbol{\mu}_{a}=\left(\mu_{1}, \mu_{2}\right)^{T}$ and

$$
\begin{aligned}
\sigma_{a, 1}^{2} & =\sigma_{1}^{2}\left[\frac{\left(1+\delta^{2}-2 \Delta \delta\right)\left(1-\rho^{2}\right)}{\left(1+\delta^{2}-2 \Delta \delta\right)-(1-\Delta \delta)^{2} \rho^{2}}\right], \\
\sigma_{a, 2}^{2} & =\sigma_{2}^{2}\left[\frac{\left(1-\delta^{2}\right)^{2}\left(1-\rho^{2}\right)}{\left(1+\delta^{2}-2 \Delta \delta\right)-(1-\Delta \delta)^{2} \rho^{2}}\right], \\
\rho_{a} & =\rho\left[\frac{\left.\left(1-\delta^{2}\right)^{2}(1-\Delta \delta)\left(1-\rho^{2}\right)\right)}{\left(1+\delta^{2}-2 \Delta \delta\right)-(1-\Delta \delta)^{2} \rho^{2}}\right] \frac{\sigma_{1} \sigma_{2}}{\sigma_{a, 1} \sigma_{a, 2}} .
\end{aligned}
$$


Corollary 4.4 shows the combined impact of the risk-tolerance and confidence/doubt on the market is rather complicated. Note that, if $\delta=0$, that is there is no biased belief in the standard deviation, the consensus belief is reduced to the benchmark belief. Jouni and Napp (2006) argue that a positive correlation between risk tolerance and doubt can help to resolve the puzzles. To illustrate the impact, we choose $\Delta=-0.2$ and $\delta=0.2$ so that $\delta \Delta<0$, that is the more risk-tolerant agent is also doubtful. We report the numerical results in Tab 4.1 for Case 4 . We can see that the market risk premium increases and risk-free rate reduces, but the magnitudes of the changes are not very significant to resolve the puzzles. Consisting with the survey result in Giordani and Soderlind (2006), this illustrates that doubt is not a promising explanation of the equity premium puzzle.

4.5. Impact of the Existence of a Risk-free Asset. In all of the above cases, we have assumed that there exists a risk-free security in the market, which is in netzero supply to allow investors borrow and lend at the risk-free rate. According to equation (4.11) and (2.2), this implies that both investors have the same CER, that is $\lambda_{i}^{*}=R_{f}$ for $i=1,2$. If there is no risk-free security in the economy, then obviously investors would have different CER and the aggregate market's CER is risk-tolerance weighted average of investors' CER. Note that when investors have common variance/covaraince matrices, then adding a risk-free security to the market would not change the consensus belief or the market equilibrium. This is, when $V_{i}=V_{o}$, the consensus belief of expected asset return are identical with or without a risk-free asset and therefore we have

$$
\lambda_{a}^{*}=R_{f}=\frac{\mathbf{1}^{T} V_{a}^{-1} \mu_{a}-\frac{1}{\tau_{a}}}{\mathbf{1}^{T} V_{a}^{-1} \mathbf{1}}
$$

Hence, the aggregate market's CER would not be affected by the existence of a riskfree security which is in net-zero supply. However, this is no longer the case when investor have different beliefs about the varaiance/covariances of asset return. Market could arrive at a different equilibrium with or without a risk-free security.

To illustrate the impact of the existence of the risk-less asset, we consider the Case 3 , which is the most promising case to resolve the puzzles, but assume that the market does not have a risk-free asset. Because of the complexity, the impact is examined by conducting numerical analysis.

In Figure (4.2) and Table 4.2, subscripts $(f)$ is referring to the situation where there exists a risk-free security in the market and subscript $(z)$ is referring the case where no risk-free borrowing or lending are allowed. Both Figure (4.2) and the numerical results in Table (4.2) show that the existence of the risk-free magnifies the effect of heterogeneity on the market. Without the risk-free security, the market portfolio consists much less of the riskier asset (asset 2). As the result, the CER, market expected return and market volatility are all reduced significantly. Most importantly, the Sharpe ratio 


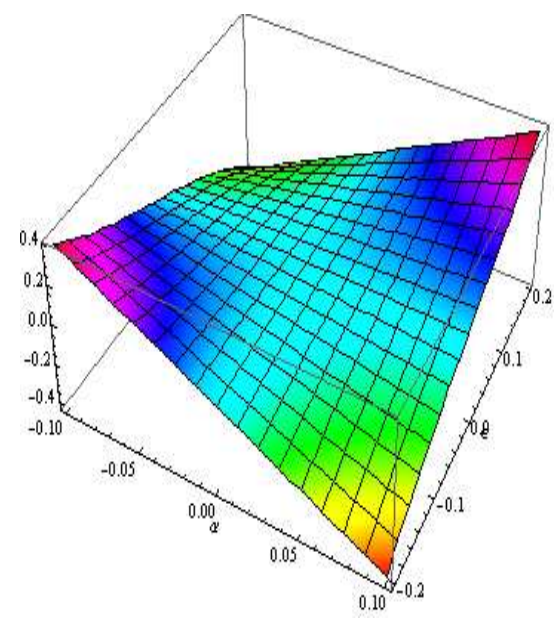

(d1) $\pi_{m, 2}^{(f)}-\pi_{m, 2}^{(z)}$

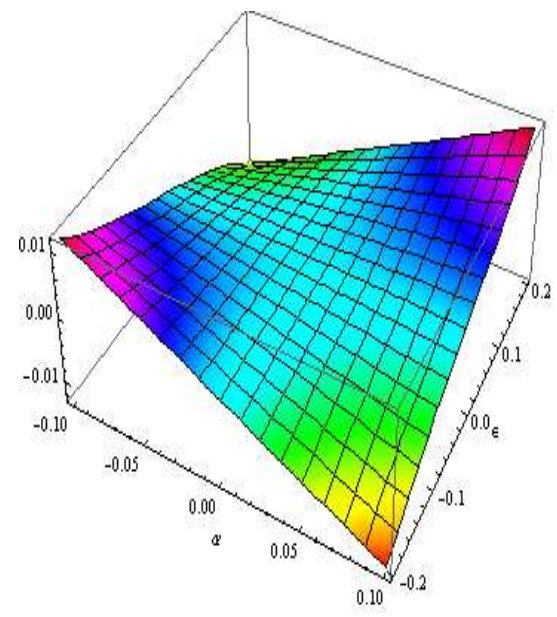

(d3) $\mathbb{E}^{(f)}\left(\tilde{r}_{m}\right)-\mathbb{E}^{(z)}\left(\tilde{r}_{m}\right)$

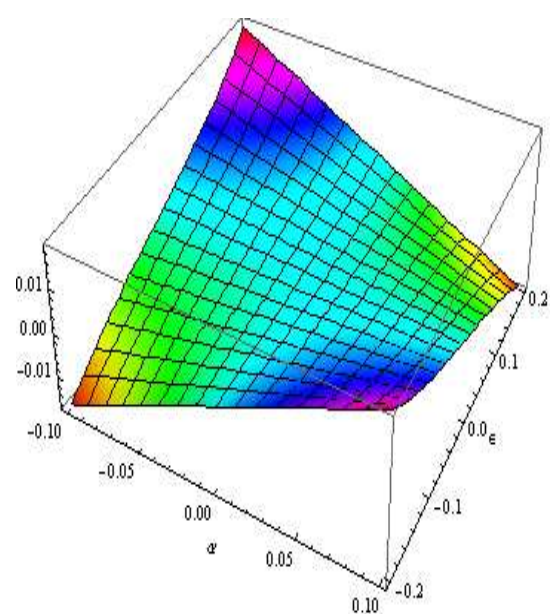

(d2) $R_{f}-\lambda_{a}^{*}$

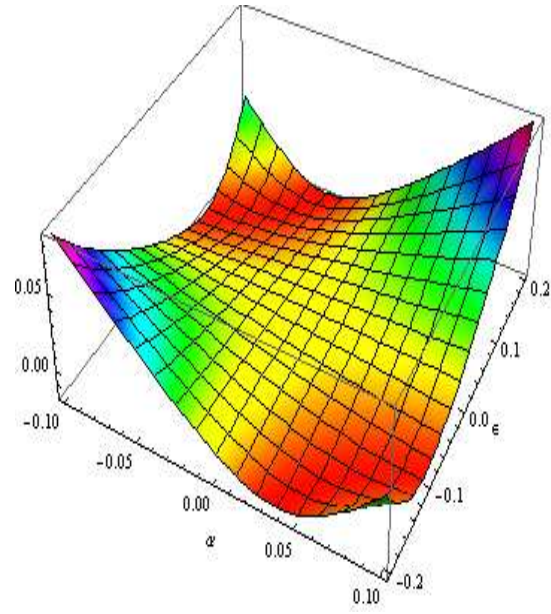

(d4) $\sigma_{m}^{(f)}-\sigma_{m}^{(z)}$

FIGURE 4.2. Impact of the existence of a risk-free security on the market proportion of asset 2, CER, market expected and volatility

\begin{tabular}{|c|c|c|c|c|}
\hline$\pi_{m, 2}^{(f)}-\pi_{m, 2}^{(z)}$ & $R_{f}-\lambda_{a}^{*}$ & $\mathbb{E}^{(f)}\left(\tilde{r}_{m}\right)-\mathbb{E}^{(z)}\left(\tilde{r}_{m}\right)$ & $\sigma_{m}^{(f)}-\sigma_{m}^{(z)}$ & $\frac{\mathbb{E}^{(f)}\left(\tilde{r}_{m}\right)-r_{f}}{\sigma_{m}^{(f)}}-\frac{\mathbb{E}^{(z)}\left(\tilde{r}_{m}\right)-\left(\lambda_{a}^{*}-1\right)}{\sigma_{m}^{(z)}}$ \\
\hline 0.3852 & $1.69 \%$ & $1.16 \%$ & $8.43 \%$ & 0.0338 \\
\hline
\end{tabular}

TABLE 4.2. Impact of the existence of a risk-free security on the market proportion of asset $2 \pi_{m, 2}^{(f)}-\pi_{m, 2}^{(z)}$, CER $R_{f}-\lambda_{a}^{*}$, market expected return $\mathbb{E}^{(f)}\left(\tilde{r}_{m}\right)-\mathbb{E}^{(z)}\left(\tilde{r}_{m}\right)$, volatility $\sigma_{m}^{(f)}-\sigma_{m}^{(z)}$ and Sharpe Ratio $\frac{\mathbb{E}\left(\tilde{r}_{m}\right)-r_{f}}{\sigma_{m}}-\frac{\mathbb{E}\left(\tilde{r}_{m}\right)-\left(\lambda_{a}^{*}-1\right)}{\sigma_{m}}$

also decreases ${ }^{5}$. Hence market can benefit from the existence of a risk-free security in terms of mean-variance efficiency, although it is in net-zero supply. The intuition is

${ }^{5}$ In the case without a risk-free security, we use $\lambda_{a}^{*}-1$ which is the expected return of the minimumvariance zero-beta portfolio under the consensus belief in calculating the Sharpe ratio. 
that, without a risk-free security, investors are restricted from taking positions to fully reflect their beliefs. Thus, the effect of heterogeneity on market equilibrium is also reduced.

4.6. Biased Beliefs in the "Safe" Stock. In the previous cases, agents are assume to have disagreement over the distribution of the terminal return of the "risky" stock, in the sense that the stock has a higher expected return and higher risk. The question is that: what are the impact of the heterogeneous beliefs in the stock with lower expected return and lower risk. We now consider this scenario in which agents have heterogeneous belief regarding distribution of terminal return of the "safe" asset, which has a less uncertain terminal return with lower expected value in the benchmark belief. The impact is illustrated by considering the following example.

Example 4.5. Let the two risky assets ${ }^{6}$ in the economy have expected returns $\left(\mu_{1}, \mu_{2}\right)=$ $(1.09,1.06)$ and standard deviations $\left(\sigma_{1}, \sigma_{2}\right)=(0.3,0.08)$ and correlation coefficient $\rho=0.8$. Agents have heterogeneous beliefs about terminal return of asset 2 , and different risk tolerance. Their heterogeneity is characterized by parameters $\Delta, \delta, \varepsilon$ and $\alpha$ as described earlier in this section.

\begin{tabular}{|c|c|c|c|c|c|c|}
\hline Cases & $(\Delta, \delta, \varepsilon, \alpha)$ & $\pi_{m, 2}$ & $\sigma\left(\tilde{r}_{m}\right)$ & $\mathbb{E}\left(\tilde{r}_{m}-r_{f}\right)$ & $r_{f}$ & $\frac{\mathbb{E}\left(\tilde{r}_{m}\right)-r_{f}}{\sigma_{m}}$ \\
\hline Benchmark & $(0,0,0,0)$ & 0.038 & $8.63 \%$ & $1.49 \%$ & $4.62 \%$ & 0.1727 \\
\hline Case 1 & $(-0.2,0,0,0.1)$ & 0.2207 & $12.2 \%$ & $4.63 \%$ & $2.04 \%$ & 0.3794 \\
\hline Case 2 & $(0,0.2,0,0.1)$ & 0.3854 & $12.99 \%$ & $6.11 \%$ & $1.04 \%$ & 0.4710 \\
\hline Case 3 & $(0,0,-0.2,0.1)$ & 0.2525 & $10.11 \%$ & $1.87 \%$ & $4.9 \%$ & 0.1846 \\
\hline Case 4 & $(0.2,0.2,0,0)$ & 0.0637 & $9.09 \%$ & $1.48 \%$ & $4.71 \%$ & 0.1633 \\
\hline
\end{tabular}

TABLE 4.3. Effects of heterogeneity on the market proportion of asset $2\left(\pi_{m, 2}\right)$, market volatility $\left(\sigma\left(\tilde{r}_{m}\right)\right)$, market risk-premium $\left(\mathbb{E}\left(\tilde{r}_{m}-r_{f}\right)\right)$, the risk-free rate $\left(r_{f}\right)$ and the Sharpe ratio $\frac{\mathbb{E}\left(\tilde{r}_{m}\right)-r_{f}}{\sigma_{m}}$ for the three cases, compared with the benchmark homogeneous case.

We redo the numerical analysis in Table 4.1 for the four cases and present the results in Table 4.3. The interesting cases (i) Case 1 when $(\Delta, \delta, \varepsilon, \alpha)=(-0.2,0,0,0.1)$ and (ii) Case 2 when $(\Delta, \delta, \varepsilon, \alpha)=(0,0.2,0,0.1)$. In both the cases, there is a significant increase in market risk premium and reduction in risk-free rate. Now we provide some explanations for these results. For (i), we have $\alpha \Delta<0$, suggesting that there is a positive correlation between the risk-tolerant and pessimism. This leads the aggregate market to perceiving a lower expected return for asset 2 (see (4.2)), therefore investing more into asset 1 (risky stock), driving up the aggregate market expected return and volatility. However, in contrast with Case 1 in Table 4.1, here the risk-free also

${ }^{6}$ Basically, we swap the two risky assets and still consider the biased beliefs in the second asset. 
reduces significantly because the market is pessimistic about expected equity returns overall (whereas in Case 1, market was optimistic about expected equity returns), thus more willing to invest in the risk-free security. In (ii), the aggregate market becomes pessimistic about future return for both asset (see (4.12)) since $\alpha \delta>0$ (the more optimistic agent is less confident). As a result, the market is even more willing to invest in the risk-free security than in the case (i), thus the reduction in risk-free rate is greater in this case. Therefore, the combined effect of heterogeneity in the beliefs of the expected and variance of terminal return leads to the most desirable result of a low risk-free rate and high market risk premium. This case also produces the highest Sharpe ratio amongst all the cases considered.

\section{CONCLUSION}

Heterogeneity, reflecting diversity and disagreement, among investors in financial markets is very common and it has significant impact on the market. Within the standard mean variance framework, we examine the impact of the heterogeneity, in particular the risk tolerance, optimism/pessimism and confidence/dobut, on the market equilibrium. To make an explicit analysis, we consider a market with two heterogeneous agents, two risky assets and a risk-free asset, in which agents have different risk tolerance and agree on the expected return and standard deviation of one asset but not the other. Agents can also be biased on the return correlation. By assuming heterogeneity and bounded rationality of investors, we characterize the market equilibrium through a consensus belief and the boundedly rational equilibrium.

By considering mean-preserved spreads in agents' preference and different aspects of the biased beliefs, we obtain some analytical results on the joint impact of the risk tolerance, optimism/pessimism, and confidence/doubt on the market equilibrium, risk premium of the risky assets, market portfolio, and the risk-free rate. We derive the CAPM relation under the heterogeneous beliefs, in which the heterogeneity becomes a risk factor of the systematic risk of the risky assets. However, this risk factor can be either positive or negative, depending on the correlations among the risk tolerance, optimism/pessimism, and confidence/doubt, and the nature of the stock on which agents disagree. We show that on the one hand, when agents disagree on and have biased beliefs in the risky stock, positive correlations between either risk tolerance and optimism/doubt or pessimism and doubt can explain the equity premium and risk-free rate puzzles, but the amount of pessimism and doubt provides only a rather small improvement in the puzzles, which is consistent with the survey data. However, when the optimistic agent believes in higher return correlation (comparing with the pessimistic agent believes in lower return correlation), it provides the most promising explanation on the puzzles and the amount of the changes in resolving the puzzles are significant. This clearly indicates the important role played by the disagreement in the asset return 
correlation, which has not been explored in the literature. On the other hand, when agents disagree on and have biased beliefs in the safe stock, positive correlations between either the risk tolerance and optimism or pessimism and doubt provide the most promising explanations on the puzzles. In particular, the amount of pessimism and doubt provides a significant improvement in the puzzles. In this case, the biased belief in the return correlation provides little explanation on the puzzles.

The biased beliefs may depend on the market conditions. Intuitively, there may be more disagreement on the risky stocks when markets are moving upwards and on the safe stocks when markets are moving downwards. The empirical implications of the results obtained in this paper would be of very interesting. The disagreement in this paper is characterized by mean preserved spreads about a benchmark homogeneous belief. It would also be interesting to extend the analysis to situations with skewed distribution about the heterogeneous beliefs such as in Abel (2002). In addition, extension to a dynamical model to examine the profitability and survivability of agents with different beliefs and the impact on the market would be interesting. We leave these to future research.

\section{REFERENCES}

Abel, A. (1989), Asset prices under heterogeneous beliefs: Implications for the equity premium, working paper 09-89, Rodney L. White Center for Financial Research.

Abel, A. (2002), 'An exploration of the effects of pessimism and doubt on asset returns', Journal of Economic Dynamics and Control 26, 1075-1092.

Barberis, N., Huang, M. and Santos, T. (2001), 'Prospect theory and asset prices', Quarterly Journal of Economics 116, 1-53.

Benartzi, S. and Thaler, R. (1995), 'Myopic loss aversion and the equity premium puzzle', Quarterly Jounral of Economics 100, 73-92.

Böhm, V. and Chiarella, C. (2005), 'Mean variance preferences, expectations formation, and the dynamics of random asset prices', Mathematical Finance 15, 61-97.

Böhm, V. and Wenzelburger, J. (2005), 'On the performance of efficient portfolios', Journal of Economic Dynamics and Control 29, 721-740.

Brunnermeier, M. and Parker, J. (2005), 'Optimal expectations', American Economic Review 94, 10921118.

Calvet, L., Grandmont, J.-M. and Lemaire, I. (2004), Aggregation of Heterogeneous beliefs and asset pricing in complete financial markets, working paper 2004-12, CREST.

Chiarella, C., Dieci, R. and He, X. (2006), Aggregation of Heterogeneous beliefs and asset pricing theory: A mean-variance analysis, technical report 186, Quantitative Finance Research Center, University of Technology, Sydney.

Chiarella, C., Dieci, R. and He, X. (2009), A framework for CAPM with heterogeneous beliefs, Springer, p. in press. in Nonlinear Dynamics in Economics, Finance and Social Sciences: Essays in Honour of John Barkley Rosser Jr., Eds. Bischi, G.-I., C. Chiarella and L. Gardini.

Daniel, K., Hirshleifer, D. and Subrahmanyam, A. (1998), 'A theory of overconfidence, self-attribution, and security market under- and over-reactions', Journal of Finance 53, 1839-1885. 
DeLong, J., Shleifer, A., Summers, L. and Waldmann, R. (1990), 'Noise trader risk in financial markets', Journal of Political Economy 98, 703-738.

Detemple, J. and Murthy, S. (1994), 'Intertemporal asset pricing with heterogeneous beliefs', Journal of Economic Theory 62, 294-320.

Giordani, P. and Soderlind, P. (2006), 'Is thee evidence of pessimism and doubt in subjective distribution? implications for the equity premium puzzle', Journal of Economic Dynamics and Control 30, 1027-1043.

Gollier, C. (2007), 'Whom should we believe? aggregation of heterogeneous beliefs', Journal of Risk and Uncertainty 35, 107-127.

He, X. and Shi, L. (2009), Portfolio analysis and Zero-Beta CAPM with Heterogeneous beliefs, Technical Report 244, Quantitative Finance Research Center, University of Technology, Sydney.

Hirshleifer, D. (2001), 'Investor psychology and asset pricing', Journal of Finance 64, 1533-1597.

Horst, U. and Wenzelburger, J. (2008), 'On no-ergodic asset prices', Economic Theory 34(2), 207-234.

Hvide, H. K. (2002), 'Pragmatic beliefs and overconfidence', Journal of Economic Behavior and Organization 48, 15-28.

Jouini, E. and Napp, C. (2006), 'Heterogeneous beliefs and asset pricing in discrete time: An analysis of pessimism and doubt', Journal of Economic Dynamics and Control 30, 1233-1260.

Jouini, E. and Napp, C. (2009), 'Optimal strategic beliefs', SSRN Working Paper (1362427).

Kyle, A. (1989), 'Informed speculation with imperfect competition', The Review of Economic Studies 56(3), 317-355.

Kyle, A. and Wang, A. (1997), 'Speculation duopoly with agreement to disagree: Can overconfidence survive the market test?', Journal of Finance 52, 2073-2090.

Levy, H. and Markowitz, H. (1979), 'Approximating expected utility by a function of mean and variance', American Economic Review 69(3), 308-317.

Lintner, J. (1969), 'The aggregation of investor's diverse judgements and preferences in purely competitive security markets', Journal of Financial and Quantitative Analysis 4, 347-400.

Mehra, R. and Prescott, E. (1985), 'The equity primium: a puzzle', Journal of Monetary Economics 15(2), 145-161.

Rabin, M. (1998), 'Psyschology and economics', Journal of Economic Literature 36, 11-46.

Rubinstein, M. (1976), 'The strong case for the generalized logarithmic utility model as the premier model of financial markets', Journal of Finance 31, 551-571.

Sharpe, W. (1991), 'Capital asset prices with and without negative holdings', Journal of Finance 66, 119-138.

Sharpe, W. (2007), Investors and Markets, Portfolio Choice, Asset Prices, and Investment Advice, Princeton.

Weil, P. (1989), 'The equity premium puzzle and the tisk-free rate puzzle', Journal of Monetary Economics 24, 401-421.

Wenzelburger, J. (2004), 'Learning to predict rationally when beliefs are Heterogeneous', Journal of Economic Dynamics and Control 28, 2075-2104.

Williams, J. (1977), 'Capital asset prices with Heterogeneous beliefs', Journal of Financial Economics 5, 219-239.

Zapatero, F. (1998), 'Effects of financial innovations on market volatility when beliefs are heterogeneous', Journal of Economic Dynamics and Control 22, 597-626. 